\title{
DYNAMICS OF STAR CLUSTERS : COMPARISON OF THEORY WITH OBSERVATIONS AND SIMULATIONS
}

\author{
R. WIELEN \\ Astronomisches Rechen-Institut, Heidelberg, \\ and \\ Hamburger Sternwarte, Hamburg-Bergedorf, Germany
}

\begin{abstract}
Stellar dynamical theories for collisional systems are tested by numerical $N$-body simulations of isolated star clusters with $N$ up to 500 . For the dynamical evolution of the density distribution in star clusters, good agreement is found between Monte Carlo results and $N$-body models. There remains a discrepancy between theory and simulations in the rate of stellar escape from clusters and/or in the mechanism by which escapers are produced. Simulations of non-isolated star clusters are compared with observations of open clusters. The observed age distribution of open clusters can be explained by the dynamical dissolution of clusters. Stars of low mass do not escape relatively more frequently than stars of average mass.
\end{abstract}

\section{Introduction}

In stellar dynamics, we have essentially three categories of knowledge: observations, simulations and theories. Observations deal with stellar systems actually in the sky. Theories aim at a mathematical description of stellar systems on the basis of plausible physical and statistical assumptions. Simulations are numerical experiments in which we use directly the basic law of gravitation without any other a priori assumption. When we try to compare the results of these three approaches in order to understand stellar systems, it is easiest to compare theories and simulations, because we have rather complete control over the objects under investigation. For such a comparison of theory versus simulation, models of stellar systems should be used which are as simple as possible in order to test the basic assumptions of a theory. For example, isolated systems with stars of equal mass can be investigated first. Only if the theory has successfully passed such a test, should more realistic situations be studied. In a comparison of numerical simulations with astronomical observations, the models must be as realistic as possible. We can then test whether we have included in our simulations all the relevant physical phenomena (external forces, mass loss of stars etc.) and whether we have used realistic values for the parameters of the model (e.g. for its total mass). A direct comparison of a simplified version of a theory with observations is usually more difficult to judge in its implications. This is especially true if such a theory is in conflict with the results of simulations, but nevertheless is able to reproduce some observed properties of actual stellar systems.

In this review, we shall consider only stellar systems in which encounters among the stars are important ('collisional systems'). Open star clusters are typical examples of such systems. Since a number of review papers on the dynamical evolution of 
collisional systems have been published recently (Aarseth, 1973; Hénon, 1973; King, 1974; Wielen, 1974), we shall concentrate here on recent results. Comparisons between theory and simulation and between observations and simulations will be presented. For a comparison between theory and observations, we refer to King (1975) for globular clusters and to Prata (1971) for the rich open cluster M67.

\section{Comparison of Theory with Simulations}

In this section, we discuss isolated star clusters, containing stars of equal mass or stars with a realistic mass spectrum. First, we compare the results of theory and simulations for the evolution of the spatial structure of a cluster. Second, the results on the escape of stars from a cluster will be compared.

In the Figures 1 and 2 (Aarseth et al., 1974), we compare results for the evolution of the spatial structure of a cluster by plotting the radii of spheres which contain $10 \%, 50 \%$ and $90 \%$ of the total mass as a function of time. The unit of time corresponds to about 11 relaxation times $t_{r h}$, defined by Spitzer and Hart (1971). In all the calculations, the Plummer model has been adopted as a common initial state of the cluster.

Figure 1 shows the results for the equal-mass case. In the $N$-body simulations by Aarseth (1974) and by Wielen (1974), the dynamical evolution of the cluster has been followed by integrating the complete equations of motion of the $N$-body problem for $N=250$ or 100 stars. The Monte Carlo results obtained by Hénon (1973) and by Shull and Spitzer (1974) rely on the classical theory of relaxation. The cumulative effect of weak two-body encounters is described by the Fokker-Planck equation which is solved by a numerical Monte Carlo procedure. The fluid-dynamical approach of Larson (1970) is also based on the Fokker-Planck equation, but further approximations are introduced in order to simplify the numerical treatment. The general shape of the theoretical and experimental curves is quite similar. A slight discrepancy in the time scale of evolution is indicated: The Monte Carlo models evolve faster than the $N$-body models by a factor of about 1.5 , and the fluid-dynamical model by a factor of 2 or 3 . After about 1.5 units of time, the central density becomes infinite according to the theoretical models. At about the same time, a close central binary forms near the center in the $N$-body models.

In Figure 2, we present results for the case of a realistic distribution of stellar masses listed in Table III. Six $N$-body models (Wielen, 1974) with $N$ up to 500 stars are compared with three new Monte Carlo models obtained by Hénon. Again, we find a nice qualitative agreement between theory and simulations. In the case of unequal masses, the Monte Carlo models evolve faster than the $N$-body models by a factor of at least 2. Also the central collapse of the Monte Carlo models occurs earlier $(t \sim 0.15)$ than the formation of a close central binary in the $N$-body models $(t \sim 0.5)$. A preliminary comparison of the segregation of stars of different masses suggests good agreement between the Monte Carlo computations and the $N$-body models in this respect.

From the comparisons shown in Figures 1 and 2, we conclude that the classical theory of relaxation, if used in a realistic form such as the Monte Carlo scheme, is 


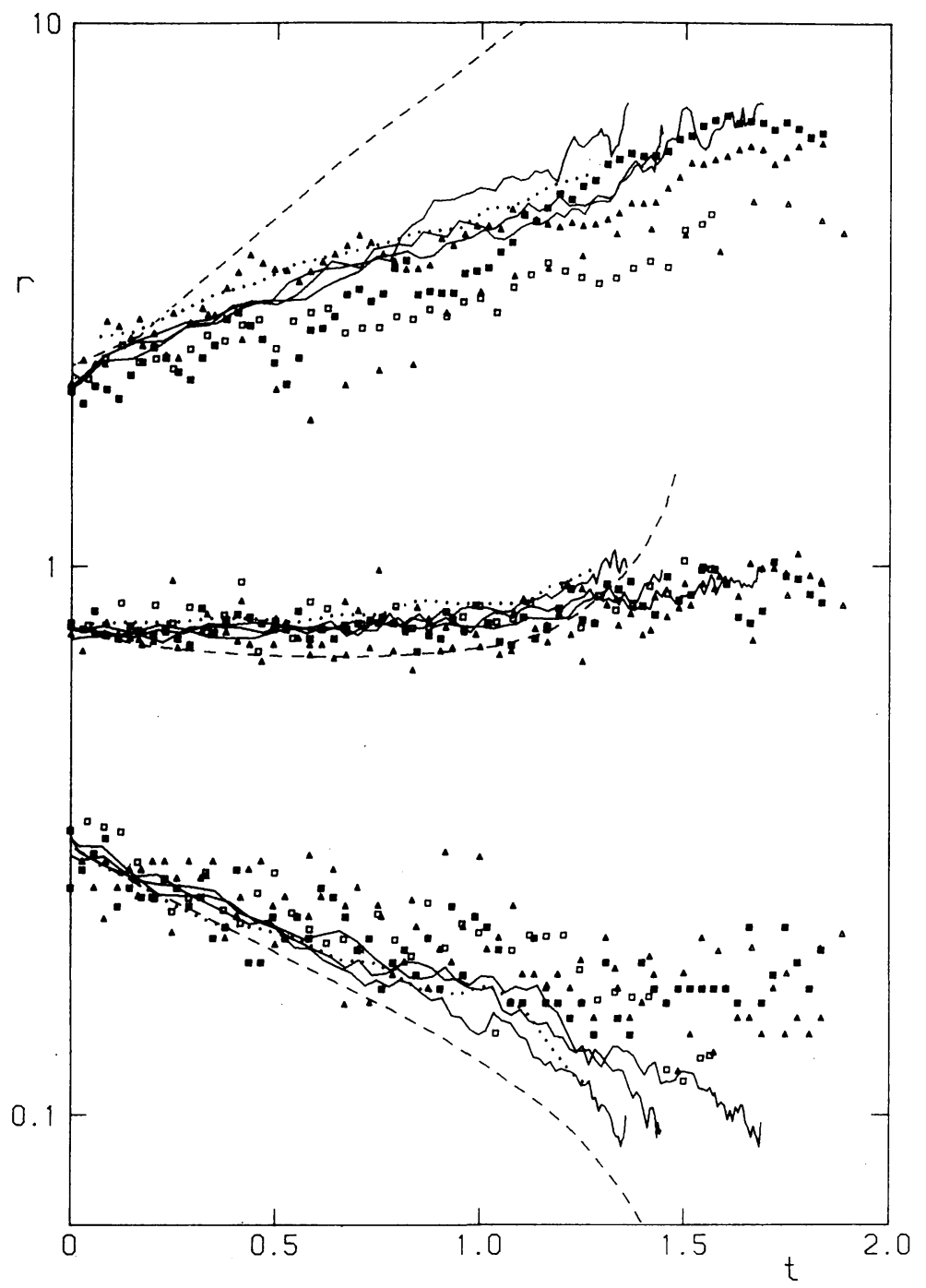

Fig. 1. Radii $r$ of spheres containing $10 \%, 50 \%$ and $90 \%$ of the total mass, as a function of time $t$, for a cluster containing stars of equal masses. $N$-body simulations by Aarseth $(N=250$, filled symbols) and by Wielen ( $N=250$, open squares; $N=100$, open triangles). Monte Carlo models by Hénon (solid lines) and by Shull and Spitzer (dotted lines). Fluid-dynamical results by Larson (dashed lines).

able to predict rather successfully the evolution of the spatial structure of star clusters observed in $\mathrm{N}$-body simulations. This confirms the basic assumption of the theory, namely that the diffusion effect of independent, weak two-body encounters is the main source of relaxation in star clusters. The discrepancy between theory and simulations regarding the time scale of the evolution can be removed by a proper modification of the theory (Hénon, 1975). The comparison indicates furthermore that the range of validity of $N$-body simulations and Monte Carlo computations 


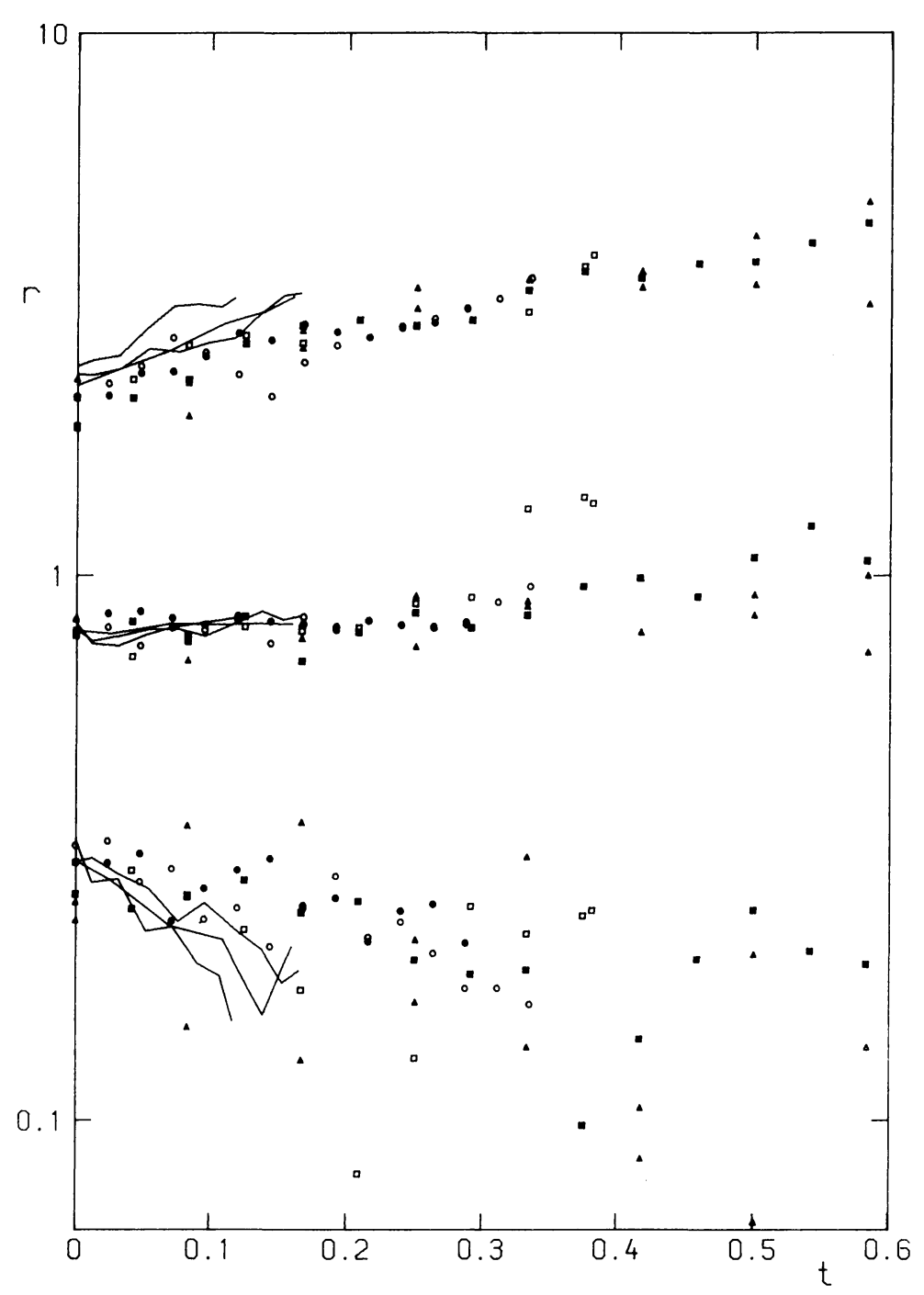

Fig. 2. The same quantities as shown in Figure 1, but for a cluster containing stars with a realistic mass spectrum. $N$-body simulations by Wielen: $N=100$ (triangles), $N=250$ (squares), $N=500$ (circles). Monte Carlo models by Hénon (solid lines).

overlaps for $N \sim 100$ to 500 stars. Hence the $N$-body results for $N \leqslant 500$ may now be extrapolated to much higher values of $N$ with some confidence.

Beside the positive conclusions, there remain some problems. It is unclear whether the dominant central binary usually formed in $N$-body integrations can be essentially identified with the infinite central density predicted by the theory. Even within the frame of the theory itself, the central collapse can be interpreted in different ways: Spitzer advocates that a finite fraction $(\sim 10 \%)$ of the total mass of a cluster is involved in the collapse; in Hénon's picture, the mass of the collapsing core becomes 
zero when the central density becomes infinite. It is not an easy task to continue the Monte Carlo scheme beyond the time of the central collapse. Recently, Hénon (1975) has proposed a way of extending the Monte Carlo computations past the collapse time by introducing an artificial 'well' which absorbs the negative energy flowing towards the center. As we shall discuss below, the Monte Carlo method does not seem to reproduce correctly the escape of stars. Both difficulties, the handling of the collapsed core and the escape of stars, cast some doubts on whether the theory in the present form is able to describe accurately the long-term evolution and the final dissolution of star clusters.

The $N$-body simulations give rather detailed information on the mechanism by which stars can gain enough energy to escape from the cluster. The classical theories predict a slow diffusion of stars towards positive total energy due to distant encounters. Hénon $(1960,1969)$, however, has advocated that in isolated clusters escapers are mainly produced by single, close two-body encounters. The $N$-body models show that the total energy of an escaping star always jumps suddenly to a positive .value, and that typical escapers are strongly bound to the cluster before they gain the energy for escape (see e.g. Figures 4 and 5 in Wielen, 1974). The basic mechanism for this sudden change of the energy is in most cases a close encounter of the star which escapes afterwards, either with another single star or with a binary or a small group of stars in the core of the cluster. A detailed description and statistics on such 'escape events' have been given by Aarseth (1974). Hence the $N$-body models favor Hénon's theory of escape and contradict the classical picture of diffusion as a mechanism for escape of stars. It may be argued that the diffusion process becomes more important for rich star clusters, because the theoretically predicted ratio between the frequency of distant and close encounters is proportional to $\log N$. However, even if $10 \%$ of the escapers were caused by diffusion for $N=500$ stars, the expected contribution of distant encounters would remain small also for globular clusters with $N \sim 2 \times 10^{5}$, namely about $20 \%$ (Wielen, 1974).

The rate of escape, $\dot{N} T_{\text {cr }}$, defined as the average number of stars which escape in one crossing time $T_{\mathrm{cr}}$, is shown in Table I for stars of equal mass, and in Table II for a realistic spectrum of stellar masses. The numbers quoted for the classical theories refer to $N=100$ stars, but increase only very slowly with $N$. The number quoted for Spitzer and Thuan (1972) refers to the average escape rate of their model E1. A comparison of the theoretical predictions with the experimental results in Table I shows that all the old classical theories overestimate the escape rate in the case of equal masses. The escape rate derived from the Monte Carlo calculations of Spitzer and Thuan (1972) is in rather good agreement with the results of $N$-body simulations (Table I). This coincidence may, however, be regarded as fortuitous as long as the difference in the basic mechanism of producing escapers remains unexplained. The converse situation occurs if we compare Hénon's theory of escape with the $N$-body models. While this theory predicts correctly the escape mechanism, it fails to reproduce the escape rates. The discrepancy is even stronger for equal masses (Table I) than for unequal masses (Table II). The escape rate predicted by Hénon may be too 
TABLE I

Escape rate for isolated clusters (Stars of equal masses)

\begin{tabular}{lll}
\hline $\begin{array}{l}\text { Number of escapers } \\
\text { per crossing time }\end{array}$ & Source \\
$\dot{N} T_{\text {cr }}$ [stars] & \\
\hline 0.70 & Spitzer (1940) & \\
0.55 & Chandrasekhar (1942) & \\
1.18 & Chandrasekhar (1943) \\
1.58 & White (1949) & \\
0.98 & Spitzer and Härm (1958) \\
0.31 & King (1958) & \\
0.4 & Larson (1970) & \\
0.14 & Spitzer and Thuan (1972) \\
0.027 & Hénon (1960) & \\
\hline & & \\
0.08 & Wielen Model HE & $N=50$ \\
0.06 & Wielen Model E & $N=100$ \\
0.13 & Wielen Model DE & $N=250$ \\
0.17 & Aarseth Model III & $N=250$ \\
0.28 & Aarseth Model VI & $N=250$ \\
0.22 & Aarseth Model VII & $N=250$ \\
& &
\end{tabular}

TABLE II

Escape rate for isolated clusters

(Realistic spectrum of stellar masses)

\begin{tabular}{lll}
\hline $\begin{array}{l}\text { Number of escapers } \\
\text { per crossing time }\end{array}$ & Source & \\
$\dot{N} T_{\text {cr }}[$ stars] & & \\
\hline 0.48 & Hénon (1969) & $N=50$ \\
\hline 1.0 & Wielen Model HP & $N=100$ \\
0.8 & Wielen Models P+P2+R & $N=250$ \\
1.1 & Wielen Models DP + DP2 & $N=250$ \\
1.1 & Aarseth Model IV & $N=500$ \\
0.6 & Wielen Models FP+FP2 & $N=500$ \\
1.6 & Aarseth Model V & \\
\hline
\end{tabular}

small for various reasons: The theory neglects multiple encounters, binaries, core formation and mass segregation. All these phenomena enhance the escape rate in actual systems.

In Table III, we compare the relative escape rates of stars of different mass. The last three columns give results of $N$-body calculations (Wielen, 1974). The models DG3 and FG3 are non-isolated clusters. The $N$-body integrations carried out by Aarseth $(1973,1974)$ are in good agreement with our results. For isolated systems, the theories predict a strong increase in the relative escape rate with decreasing stellar mass $m$, in contradiction to the $N$-body models. This discrepancy may be due to the strong mass segregation and the formation of massive binaries, which occur 
TABLE III

Relative rate of escape for stars of different masses

$\left(\Delta N_{m} / N_{m}\right) /(\Delta N / N)$

\begin{tabular}{llllllll}
\hline$\frac{m}{\langle m\rangle}$ & $N_{m}$ & $\begin{array}{l}\text { Chandrasekhar } \\
(1942)\end{array}$ & $\begin{array}{l}\text { Spitzer } \\
\text { and Härm } \\
(1958)\end{array}$ & $\begin{array}{l}\text { Hénon } \\
(1969)\end{array}$ & $\begin{array}{l}\text { Isolated } \\
\text { models } \\
N=50-500\end{array}$ & $\begin{array}{l}\text { Model } \\
\text { DG3 } \\
N=250\end{array}$ & $\begin{array}{l}\text { Model } \\
\text { FG3 } \\
N=500\end{array}$ \\
\hline 4.4 & $4 \%$ & $1 \times 10^{-8}$ & $\sim 0$ & 0.05 & $0.4:$ & $0.3:$ & $0:$ \\
2.2 & $10 \%$ & $2 \times 10^{-4}$ & $\sim 0$ & 0.25 & $0.6:$ & $0.4:$ & $0:$ \\
1.1 & $24 \%$ & 0.27 & 0.23 & 0.71 & 1.0 & 1.1 & 0.8 \\
0.55 & $62 \%$ & 1.51 & 1.52 & 1.30 & 1.1 & 1.1 & 1.3 \\
\hline
\end{tabular}

in the $\mathrm{N}$-body models but are neglected in the theories. We shall mention here that even for the more realistic, non-isolated $\mathrm{N}$-body models (such as DG3 or FG3 in Table III), the relative escape rate does not depend significantly on the stellar mass for masses below the average mass. Only more massive stars escape less frequently than indicated by the overall escape rate. The implications of $N$-body results for the relative escape rates for the luminosity function of the Hyades have been discussed by Aarseth and Woolf (1972).

\section{Comparison of Observations with Simulations}

What astronomical observations give relevant information about the dynamical evolution of star clusters? This question has been discussed during this Symposium by King (1975) for the case of globular clusters. Hence, we shall concentrate here on open clusters.

The density distribution and the segregation of stars of different masses, although observed in projection only, must contain important information on the dynamics of clusters. But these observations hardly provide any significant test for a theory at present, since essentially all the dynamical theories (old and new ones) claim a rather perfect agreement with such observations. The luminosity function as well as the frequency of binaries in a cluster may only slightly reflect the dynamical evolution of a cluster. The relative scarcity of faint dwarfs in open clusters, compared with field stars, is probably due to special physical conditions during the formation of a cluster. Most binaries must have already existed as proto-binaries. The radius of a cluster, its overall velocity dispersion, its total mass and membership have to be considered in most cases as scaling parameters, not as independent test quantities. Of course, the knowledge of such parameters is indispensable for any reliable comparison of theories or simulations with actual clusters.

For star clusters, there exists an observable quantity which is often even more accurately known than the total mass, namely the age of a cluster. The observed distribution of the ages of open clusters contains rather direct information on the total lifetimes and hence on the time scale of the dynamical evolution of these clusters. From the observed age distribution, we can deduce that $50 \%$ of newly born open 
clusters disintegrate within $2 \times 10^{8} \mathrm{yr}, 10 \%$ live longer than $5 \times 10^{8} \mathrm{yr}$ and only $2 \%$ survive over $1 \times 10^{9} \mathrm{yr}$ (Wielen, 1971). Hence the typical lifetime of an open cluster is rather short compared to the age of the Galaxy, but there exists a wide spread in the individual lifetimes. These total lifetimes of open clusters can serve as a powerful observational test of theories and simulations of the dynamical evolution of star clusters.

The dynamical evolution of open star clusters is mainly caused by the following effects: (1) relaxation by encounters among the cluster stars, (2) the gravitational tidal field of the Galaxy, (3) fluctuating gravitational fields of passing interstellar clouds, (4) mass loss of evolved massive stars in late phases of their internal evolution. The effects (1), (2) and partly (4) have been incorporated in the $N$-body simulations of open clusters carried out by Aarseth and by Wielen. Details on these $N$-body models can be found in the reviews (Aarseth, 1973; Wielen, 1974). In addition, we present here some results on a new $N$-body model, named FG3, which is similar to the models $\mathrm{G} 3$ and DG3 but contains $N=500$ stars. The range of $N$, up to 500 , now available covers most open clusters. Therefore, a rather direct comparison of the $N$-body models with open clusters is possible.

The total lifetimes of open star clusters as predicted from $N$-body simulations by Wielen, are shown in Figure 3. Since the escape rate $\dot{N}$ is rather constant during the dynamical evolution, the evaporation time $T_{\mathrm{ev}}=N / \dot{N}$ is nearly identical with the total lifetime, if we use the initial parameters such as $N_{0}, \mathfrak{M}_{0}$ and $R_{0}$ of a cluster in the applications. In Figure 3 we have plotted the evaporation time of a cluster, $T_{\text {ev }}$, as a function of its median radius in projection $R$, for clusters containing $N=100$ stars (total mass $\left.\mathfrak{M}=50 \mathfrak{M}_{\odot}\right), 250$ stars $\left(125 \mathfrak{M}_{\odot}\right)$ and 500 stars $\left(250 \mathfrak{M}_{\odot}\right)$. A detailed description of the construction of Figure 3 from the results of $N$-body simulations can be found in Wielen's review (1974). The lifetimes predicted by Aarseth (1973) are about twice as long as those obtained by Wielen. This discrepancy is due to slightly different procedures used for identifying escapers from non-isolated clusters. The difference may disappear, if the passing HI clouds are properly incorporated into the $N$-body models, since they should rapidly remove the outermost halo of a cluster to which most of the doubtful escapers belong.

Up to now, passing interstellar clouds have been neglected in the $N$-body simulations carried out by Aarseth and Wielen. The $N$-body experiments of Bouvier and Janin (1970) were done for very small systems $(N=25)$, without considering the tidal field of the Galaxy, and their results may be strongly biased by the unrealistic mean force field of the clouds present in their calculations (see my remark after Janin's paper (1975) during this Symposium). Hence, at present the effect of interstellar clouds can only be inferred from theoretical studies, especially from the Monte Carlo models of Spitzer and Chevalier. In Figure 4, the dissolution time $T_{\text {dis }}=T_{\text {sh }} / 2$ of a cluster, due to the gravitational shocks of passing clouds alone (Spitzer and Chevalier, 1973 ), is compared with the $N$-body results of Figure 3 for $N=500$ stars and $\mathfrak{M}=250$ $\mathfrak{M}_{\odot}$ as a function of the median radius $R$ of the cluster. Since the properties of interstellar clouds, e.g. their typical masses, are still very uncertain, we show in Figure 4 


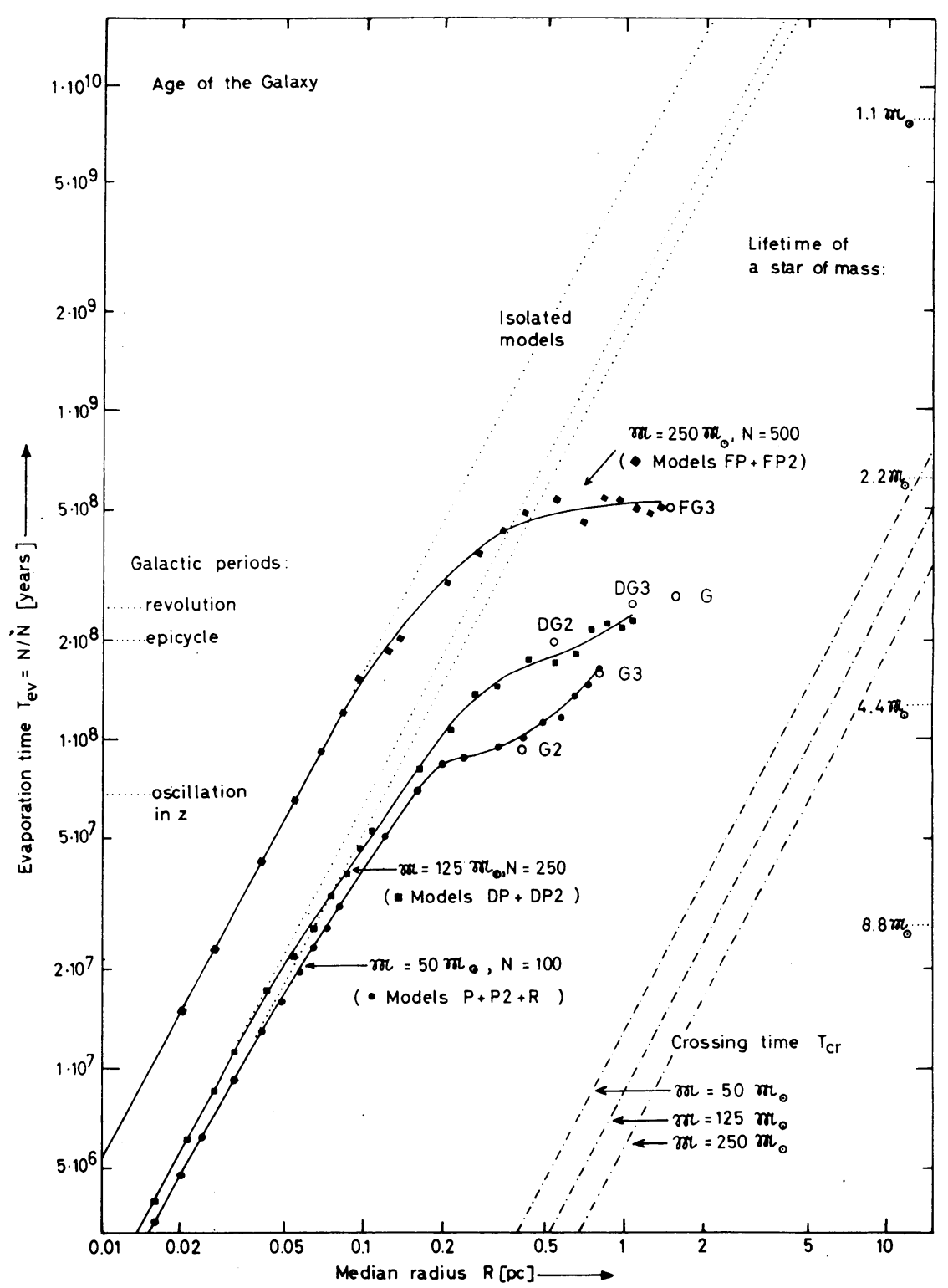

Fig. 3. Evaporation times of $N$-body models for open clusters as a function of the median radius $R$ of the projected cluster for various total masses $\mathfrak{M}$, shown by the symbols and the fitted solid lines.

two cases: For the lower line, we have adopted the shock time $T_{\mathrm{sh}}$ according to the cloud data proposed by Spitzer and Chevalier (1973), while the upper line corresponds to the 'standard clouds' described by Spitzer (1968). The dashed line which connects the $N$-body results with the theoretical line for $T_{\mathrm{sh}} / 2$, is derived by adding the corresponding escape rates, i.e. by taking the harmonic mean of $T_{\mathrm{ev}}$ and $T_{\mathrm{sh}} / 2$. Figure 4 seems to indicate that the dynamical dissolution of an open cluster is sig- 


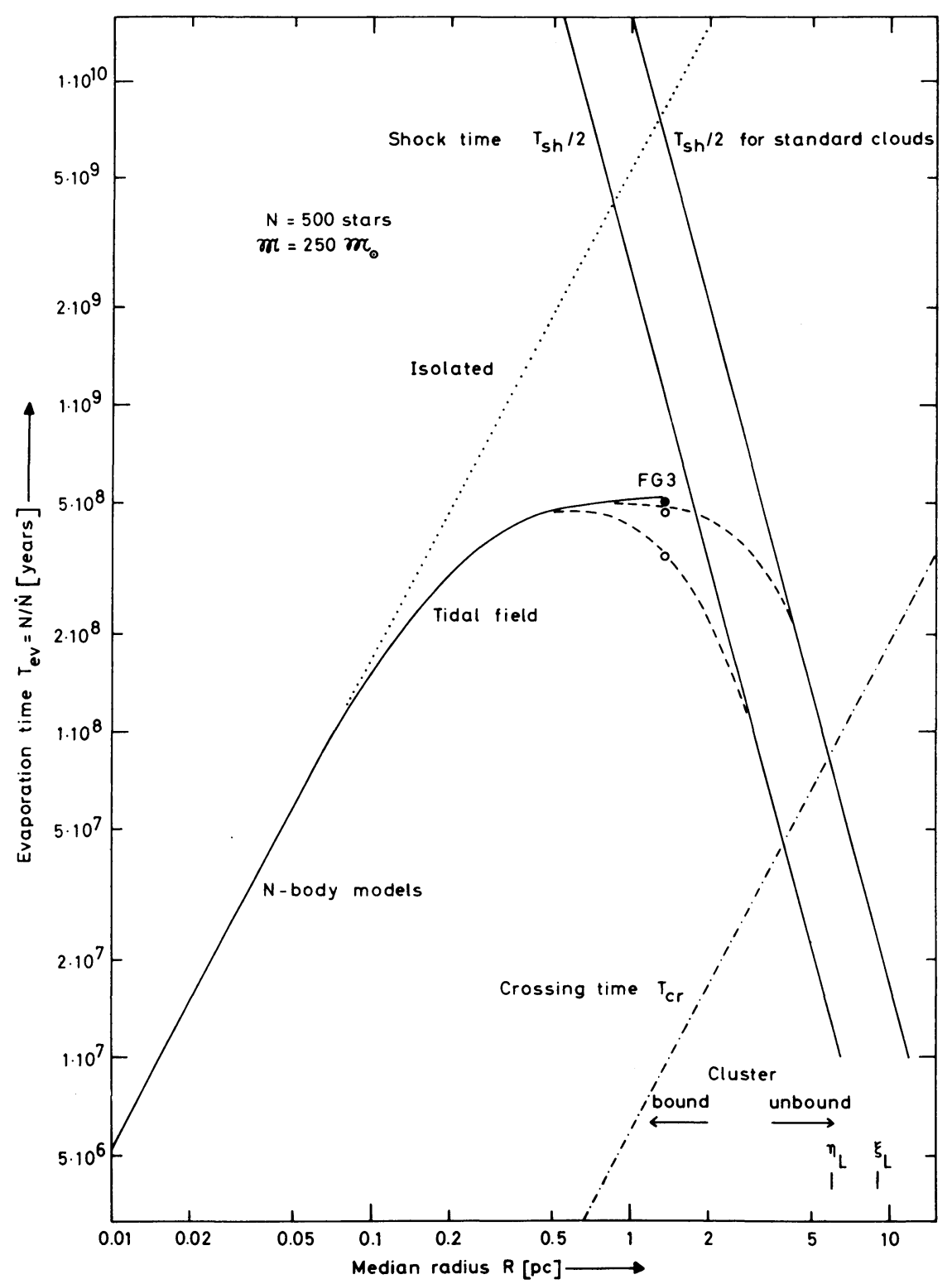

Fig. 4. Evaporation time of $N$-body models (solid curve, circles) and dissolution time by clouds, $T_{\mathrm{sh}} / 2$ (solid lines), predicted from Monte Carlo models of Spitzer and Chevalier, for a total mass of $\mathfrak{M}=250 \mathfrak{M}_{\odot}$ as a function of the median radius $R$ of a cluster.

nificantly accelerated by passing interstellar clouds only if the cluster is already either weakly bound or even unstable because of the tidal field of the Galaxy (i.e. if $R \gtrsim 2 \mathrm{pc}$ for $\mathfrak{M}=250 \mathfrak{M}_{\odot}$ ).

The shape of the outermost regions of an open cluster may help to decide obser- 
vationally whether gravitational shocks due to interstellar clouds are important. According to the $N$-body simulations without clouds, the tidal field of the Galaxy produces a significant flattening (up to 1:2) of the halo of a cluster perpendicular to the galactic plane. If the clouds are dynamically important for an open cluster, the halo may be rounder and smaller in size, because it will not fill any more the critical volume (see Figure 6 of Wielen, 1974) corresponding to the tidal radius of the cluster, since the outermost stars are rapidly stripped off from the cluster by the gravitational interaction with the clouds. Unfortunately, the presently available observations of the sparsely populated halos of open clusters, e.g. of the Hyades, do not allow any accurate statement on the degree of a probable flattening.

In order to apply the results of $N$-body simulations, we need information on the initial total masses $\mathfrak{M}$ and median radii $R$ of open clusters. From young clusters, we find that most clusters have median radii in projection between $0.5 \mathrm{pc}$ and $3 \mathrm{pc}$; $R \sim 1 \mathrm{pe}$ is quite typical. It is much more difficult to estimate the total mass of a cluster. Only for a very few open clusters can the total mass be determined by applying the virial theorem. Total masses derived from extrapolating the luminosity function in a cluster from that of field stars, are very probably too high, because of the relative underabundance of low-mass stars in open clusters (e.g. van den Bergh and Sher, 1960). Total masses of open cluster may range from less than $50 \mathfrak{M}_{\odot}$ to more than $10^{3} \mathfrak{M}_{\odot}$, but most clusters seem to have rather small values of $\mathfrak{M}$.

The observed typical total lifetime of open clusters, $2 \times 10^{8} \mathrm{yr}$, can be explained by the $N$-body models, if we accept $R \sim 1$ pc and $\mathfrak{M} \sim 100 \mathfrak{M}_{\odot}(N \sim 200$ stars $)$ as being typical for an open cluster of zero age. According to Figures 3 and 4 the lifetime is not very sensitive to variations of $R$ for $R \sim 1 \mathrm{pc}$, but increases roughly proportional to $N$ for a fixed value of the mean stellar mass $\mathfrak{M} / N$. Since a typical value of $\mathfrak{M} \sim 100$ $\mathfrak{M}_{\odot}$ is not implausible, we may claim agreement between the observed and predicted typical lifetimes of open clusters for the time being. The observed range in the lifetimes of open clusters, roughly from $10^{8}$ to $10^{10} \mathrm{yr}$, can be explained as due to the variety of total masses and median radii of open clusters. The lifetimes of the oldest open clusters, e.g. NGC 188 or M67, require an initial total mass of about $5000 \mathfrak{M}_{\odot}$ for $R \sim 2$ pc.

\section{Final Remarks}

The discussion in Section 2 shows that a comparison of theory with simulations gives significant results which improve our theoretical understanding of stellar systems. In order to facilitate such comparisons, both the investigators working in the fields of theory and numerical simulations should cooperate as closely as possible in order to provide quantities which can be directly compared.

The comparison of observations with theory or simulations is still rather disappointing, because we have so few points of contact between the two kinds of investigation. In order to facilitate comparison of observations with theoretical results, the observers should be asked to publish also their raw data as far as possible and not to mix up their observations immediately with special theoretical concep- 
tions. Much observational work, which should be especially designed for a meaningful comparison with theoretical work, remains to be done for star clusters.

\section{References}

Aarseth, S. J.: 1973, Vistas in Astronomy 15, 13.

Aarseth, S. J.: 1974, Astron. Astrophys. 35, 237.

Aarseth, S. J. and Woolf, N. J.: 1972, Astrophys. Letters 12, 159.

Aarseth, S. J., Hénon, M., and Wielen, R.: 1974, Astron. Astrophys. 37, 183.

Bergh, S. van den and Sher, D.: 1960, Publ. David Dunlap Obs. 2, 203.

Bouvier, P. and Janin, G.: 1970, Astron. Astrophys. 9, 461.

Chandrasekhar, S.: 1942, Principles of Stellar Dynamics, Chicago University Press, Chicago.

Chandrasekhar, S.: 1943, Astrophys. J. 98, 54.

Hénon, M.: 1960, Ann. Astrophys. 23, 668.

Hénon, M.: 1969, Astron. Astrophys. $2,151$.

Hénon, M.: 1973, in L. Martinet and M. Mayor (eds.), Dynamical Structure and Evolution of Stellar Systems, Swiss Society of Astronomy and Astrophysics Third Advanced Course, Geneva Observatory, p. 183.

Hénon, M.: 1975, this volume, p. 133.

Janin, G. and Haggerty, M. J.: 1975, this volume, p. 61 .

King, I. R.: 1958, Astron. J. 63, 109.

King, I. R.: 1974, Celes. Mech. 9, 349.

King, I. R.: 1975, this volume, p. 99.

Larson, R. B.: 1970, Monthly Notices Roy. Astron. Soc. 150, 93.

Prata, S. W.: 1971, Astron. J. 76, 1017.

Shull, J. M. and Spitzer, L.: 1974, private communication.

Spitzer, L.: 1940, Monthly Notices Roy. Astron. Soc. 100, 396.

Spitzer, L.: 1968, Diffuse Matter in Space, Interscience Publishers (Wiley), New York-London-SydneyToronto, p. 85.

Spitzer, L. and Chevalier, R. A.: 1973, Astrophys. J. 183, 565.

Spitzer, L. and Härm, R.: 1958, Astrophys. J. 127, 544.

Spitzer, L. and Hart, M. H.: 1971, Astrophys. J. 164, 399.

Spitzer, L. and Thuan, T. X.: 1972, Astrophys. J. 175, 31.

White, M. L.: 1949, Astrophys. J. 109, 159.

Wielen, R.: 1971, Astron. Astrophys. 13, 309.

Wielen, R.: 1974, in L. N. Mavridis (ed.), Proceedings of the First European Astronomical Meeting (Athens 1972), Springer Verlag, Berlin-Heidelberg-New York, Vol. 2, p. 326.

\section{DISCUSSION}

Larson: I was intrigued to note in your first diagram that the evolution time seems to increase systematically as you go from my model which treats a cluster as a smooth fluid, to the Monte-Carlo models which represent the system as a collection of a few thousand spherical shells, and finally to the $N$-body simulations with only a few hundred discrete mass points. Thus there seems to be a trend of increasing evolution time with increasing 'graininess' of the model simulation. Do you think that there are any effects such as the formation of binaries that might tend to retard the evolution of a system with a more grainy structure (e.g. one with smaller $N$ )? 500 .

Wielen: The $N$-body results do not indicate any systematic trend with $N$ for $N$ ranging from 100 to

Spitzer: In connection with your last slide, I would question your use of standard clouds in computing tidal shocks. Clouds of all sizes are clearly present as is seen from the statistics of observed color excesses as well as from direct photographs of cloudy regions; and it is the larger clouds that make the greatest contribution to shock heating. I believe that the consideration of standard clouds only will give too small a heating rate. 
Wielen: It would be very helpful for our understanding of the dynamics of open clusters to have more reliable data on the properties of interstellar clouds.

Pişmiş: I am interested to know a bit more about the rates of escape: Although you have shown, on one of your slides, rates of escape for numbers of mass points ranging from 10 to 500 , the results are based on different models as well as probably on different computing procedures. How sensitive are these results to the adopted models and also on the different computing procedures? How would the rate of escape vary with the varying number of mass points if computations were based on the same model and on the same computing procedure?

Wielen: The escape rate measured in terms of escaping stars per crossing time seems to be rather constant in the range of $N$ from 50 to 500 . This is in agreement with theoretical predictions. 